\title{
Bio-Informatics \& Bio-Statistics for the Third Millennium
}

\author{
Eduard Babulak* \\ National Science Foundation, USA \\ *Corresponding author: Eduard Babulak, PhD, Professor, National Science Foundation, USA.
}

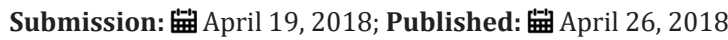

\section{Opinion}

Given the continuous increase of computation, the applied informatics biostatistics play essential role in collecting and processing enormous large amount of data generated by biological experiments, environment pollution, climate change, human health. Biostatistics is the application of statistics to a wide range of topics in biology [1]. Bioinformatics is both an umbrella term for the body of biological studies that use computer programming as part of their methodology, as well as a reference to specific analysis "pipelines" that are repeatedly used, particularly in the field of genomics [2]. The clean environment and healthy food supply to people all over the world is one of the most critical challenges for all mankind. The governments in developing countries are under enormous pressure to provide proper medical services, supply of healthy food, drinkable water, as well as creating opportunities to farm and harvest. One way to secure clean environment and to increase healthy food production both, the bio-statistics and bio-informatics are critical in creating new mathematics models and assessment of current state, as well as predicting the future states. The diversity and huge volume of collected data presents number of challenges and limitations. Most of the conventional data processing techniques are subject to standard algorithms and procedures that may require particular attributes which time dependent and nonlinear in its nature.

The Human Genome Project [3] and Large Hadron Collider Projects [4] are practical examples of utilizing biostatistics and bioinformatics tools to collect and process data, so that they may be further assessed and applied. Good understanding and effective application of biostatistics and bioinformatics fundamentals principles, contributes to creation of next generation of smart ultrapowerful computing technologies able to build a very complex and dynamic models, illustrating how the weather patterns change, how human brain works, how DNA works, and sequencing of the human genome. The application of biostatistics\& bioinformatics, have great impact on environment protection, energy production, search for new materials, and large number of industrial sectors [5].
Given the current dynamic advancements in computing and information communications technologies (ICT), the effective implementation of the Internet of Things (IoT) and computing technologies may contribute to better agriculture in way of atmosphere monitoring.

Both, the bioinformatics and biostatistics in conjunction with the advanced computing and ICT technologies, and other mathematical tools will be effectively applied to solve challenges presented by global environmental changes, and global biological systems. The way we live and the way the humanity will continue to live on our wonderful Blue Planet is in the hands of all of us. Regardless of geographical location or, age, sex, education or position that one may have, we are all equally responsible for preserving life of our children, grandchildren and many generations to come after us. Apart from technological advancement in bioinformatics and biostatistics, the political will of decision makers to protect and preserve our wonderful Blue Planet for many future generations to come is critical. The author discusses the importance of biostatistics and bioinformatics in support of live on our blue planet for many generations to come. The author promotes application of biostatistics and bioinformatics, ICT and IoT technologies in support of environment protection, modern agriculture and healthcare. The author also promotes further research and creation of global multidisciplinary research teams to build future sustainable bioinformatics solutions that will support healthy environment and food production for all mankind for many generations to come.

\section{References}

1. https://en.wikipedia.org/wiki/Biostatistics

2. https://en.wikipedia.org/wiki/Bioinformatics

3. All about the human genome project (HGP). National Human genome Research Institute.

4. The large hadron collider. CERN.

5. Research methodology and biostatistics. Michener Institute of Education at UHN. 
Creative Commons Attribution 4.0 International License

For possible submissions Click Here

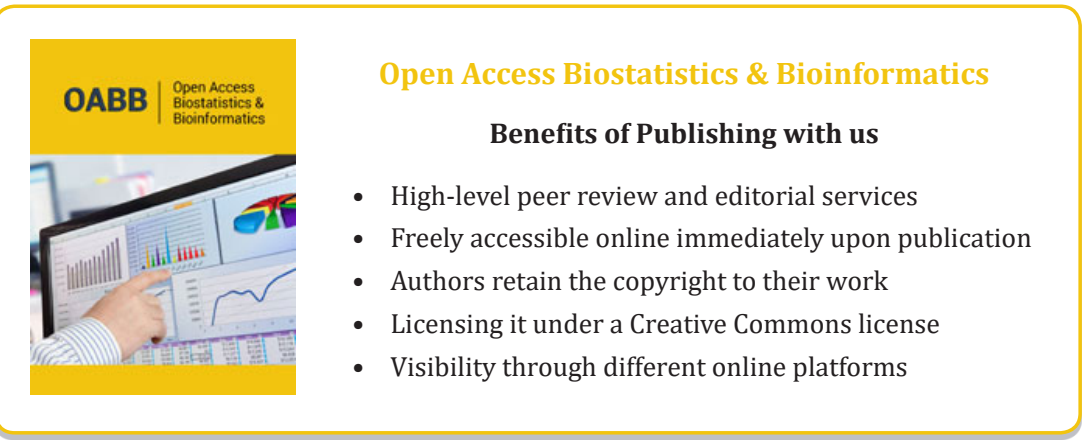

